\title{
Kernos
}

Revue internationale et pluridisciplinaire de religion grecque antique

30 | 2017

Varia

\section{Il kouros e la verità. Polivalenza delle immagini nel poema di Parmenide}

\section{Lambros Couloubaritsis}

\section{OpenEdition \\ Journals}

Édition électronique

URL : http://journals.openedition.org/kernos/2551

DOI : 10.4000/kernos.2551

ISSN : 2034-7871

Éditeur

Centre international d'étude de la religion grecque antique

Édition imprimée

Date de publication : 1 octobre 2017

Pagination : 360-364

ISSN : 0776-3824

Référence électronique

Lambros Couloubaritsis, « II kouros e la verità. Polivalenza delle immagini nel poema di Parmenide », Kernos [En ligne], 30 | 2017, mis en ligne le 01 octobre 2017, consulté le 24 septembre 2020. URL : http://journals.openedition.org/kernos/2551 ; DOI : https://doi.org/10.4000/kernos.2551

Ce document a été généré automatiquement le 24 septembre 2020.

Kernos 


\title{
Il kouros e la verità. Polivalenza delle immagini nel poema di Parmenide
}

\author{
Lambros Couloubaritsis
}

\section{RÉFÉRENCE}

Sofia RANZATO, Il kouros e la verità. Polivalenza delle immagini nel poema di Parmenide, Pisa, Edizioni ETS, 2015.1 vol. 15,5 × 22,5 cm, 292 p. (Anthropoi. Studi e materiali di Antropologia storica del mondo antico, 10). ISBN : 978-884674271-1.

1 Le critère qui régit le livre est le repérage dans le Poème de Parménide de termes, de figures et de formules qui rassemblent des images de la culture archaïque, véhiculées par des textes qui seraient connus par le public auquel il s'adresse. Cela permet à l'A. de recourir à un ensemble de documents littéraires et religieux, certains étant issus des découvertes en Grèce et en Italie du Sud dans le domaine funéraire. Elle utilise intelligemment ce dernier paramètre et accorde une importance particulière au monde infernal. Par là elle réhabilite les interprétations qui virent dans le proème une katabasis, mais en prenant comme destinataire le public, déplaçant l'intérêt vers la richesse de la culture. Le foisonnement des images révélerait la complexité à la fois de la culture de l'époque et de la pensée de Parménide ${ }^{1}$.

2 En fait, la complexité est, depuis les années 1980, au cœur de ma pensée. Dans la monographie que j'avais consacrée à Parménide, j'ai défini le mythe comme «un discours complexe à propos d'une réalité complexe où s'enchevêtrent le visible et l'invisible, et qui se déploie selon une logique qui lui est propre et en fonction d'un schème transcendantal qui unifie et régularise l'expérience ${ }^{2} »$. Mes recherches ont abouti récemment à l'étude de la complexité en tant que complexité3. La complexité se manifeste à travers les multiples interactions entre les constituants internes (les agents $\mathrm{du}$ récit) et en interaction avec le contexte, que l'on peut rendre compte par des discours 
catalogiques relativement au contexte culturel qu'elles traduisent, produisant de nouvelles propriétés et de nouvelles fonctions.

Or, ce que l'A. qualifie de "complexité » culturelle ou parménidienne concerne la profusion des images variées que le public assimilerait au cours de sa vie et que Parménide accumule pour marquer la différence de son propos. Cette procédure promeut un multiple hétérogène proche d'une démarche post-moderne, qui n'a pas grande chose à voir avec la complexité. Tout au plus peut-on l'envisager comme atteignant un seuil qui conduit à la complexité. Bien entendu, il ne s'agit pas ici de reprocher à Sofia Ranzato d'ignorer la théorie actuelle de la complexité - ignorée d'ailleurs par la plupart des chercheurs qui s'occupent de l'Antiquité. On peut néanmoins regretter l'absence d'une réflexion sur le statut du mythe et sur l'argumentation qui s'enracine dans la krisis parménidienne, qui déforce sa méthode axée uniquement sur le foisonnement d'images, d'après une méthode introduite par Louis Gernet.

4 Le livre se développe en quatre chapitres. Les deux premiers couvrent presque la moitié de l'ouvrage et concernent le proème et une partie de la seconde partie du Poème, tandis que les deux autres s'occupent du reste. Le premier chapitre (p. 25-55) traite de la dualité «Lumière et Nuit » dans le cadre du parcours emprunté par le jeune homme (kouros) où il affronte Dikè, avant de poursuivre son chemin vers la demeure d'une déesse, tandis que le deuxième (p. 57-123) porte l'accent sur sa rencontre avec la déesse en rapport avec le paysage mythique de l'outre-tombe. Le troisième chapitre (p. 125179), intitulé « Dans le cœur de la vérité bien arrondie », traverse la première partie du Poème et éclaire « ce qui est » (eon), identifié au réel et converti en entité divine, dans le sillage de Xénophane. Le quatrième chapitre (p. 181-226) développe la voie de la doxa dans l'éclairage de «La métamorphose de la cosmogonie » au moyen d'une tentative d'y intégrer les éléments « cosmiques» du proème dans l'ordre du monde, notamment la dualité Nuit et Jour / Lumière et la déesse.

Pour l'A., la profusion des images qui se répètent dans les trois segments du Poème produit des convergences entre le proème et la partie conclusive du Poème, grâce à la dualité Nuit et Lumière, qui s'accorderait à la dualité « ce qui est » et « ce qui n'est pas » concernant la vérité. La vérité propre à « ce qui est » (eon) est illustrée par la circularité consacrée dans la formule «le cœur de la vérité bien-arrondie ». Si bien que la dualité et la circularité constituent deux critères qui traversent le Poème et qui fondent son unité. Cette perspective dissimule la mise en intrigue narrative du Poème, qui intègre un mythe de voyage dans le proème, une série d'arguments dans la première partie et une cosmogonie dans la seconde partie. Elle s'oppose à la superposition homologique des thèmes. Il s'agit là d'un point important qui s'oppose à toute tentative de fusionner le proème et le récit cosmogonique sous prétexte qu'ils utilisent des images similaires. La polysémie, les métaphores et la narration, sous-déterminées dans ce livre, sont des critères indispensables, qui assurent à la philologie une place centrale dans l'analyse de la pensée archaïque.

6 Comme je l'ai montré en me référant à Richard Martin ${ }^{4}$, le terme mythos, bien avant d'exprimer le « récit ", signifiait « une façon de parler autorisée qui produit un effet ». On trouve encore ce sens chez Parménide lorsqu'il traduit la parole de la déesse. Quant au « récit », le monde archaïque utilise la sémantique du logos sur le mode du katalegein, qui se déploie selon plusieurs formes narratives: généalogies (anthropogonie, théogonie, cosmogonie...) et divers types de voyage - auxquels on peut ajouter les rites 
de passage et les initiations -, avant que la succession des arguments s'empare de la pratique du logos, laissant au discours mythique le champ libre pour exprimer les récits. D'autre part, au problème du mythe et du rite, il faut ajouter la différence entre eonta/ eon et onta/on, comme cela ressort du Papyrus de Derveni. Ce document, que l'A. cite une quarantaine de fois sans discerner ce point, consacre cette différence, eonta signifiant « choses qui sont dans le présent » et onta " choses qui sont », ce qui révèle que l'eon signifie « ce qui est dans le présent $»^{5}$.

7 Cette double insuffisance dans l'analyse de Sofia Ranzato ne m'empêche pas d'insister sur l'originalité et l'intérêt de son ouvrage, autour des deux itinéraires du proème. Le premier s'accomplirait sous l'égide de Hélios qui survole le monde, et illustre le cheminement du jeune homme qui explore le tout du monde terrestre, - idée soutenue par la leçon (controversée) panta astè (B 1,3) -, devenant un homme en possession d'un savoir (eidôs phôs), en se tenant loin des sentiers des mortels. Avec l'aide des Héliades, il atteint les portes des chemins de Nuit et de Jour, gardées par Dikè qui attribue les sanctions, avant qu'il franchisse le seuil et pénètre dans l'outre-monde, qui peut être compris comme appartenant au domaine de l'outre-tombe, mais où, à l'instar du défunt, il est possible d'accéder à un niveau plus profond de connaissance. Sauf que, dans le cas de Parménide, il s'agit de l'entrée d'un être vivant dans l'au-delà, où une déesse anonyme dispense un savoir supérieur. La descente aux enfers est analysée dans l'éclairage des travaux de G. Pugliese Carratelli qui avait réfléchi sur les lamelles d'or orphiques. Les lamelles font état d'instructions destinées à guider l'âme initiée aux mystères, au cours de son voyage d'outre-tombe ${ }^{6}$. L'analyse est complétée par d'autres apports, parmi lesquels figurent le Papyrus de Derveni ${ }^{7}$, le rapport à l'orphisme et au pythagorisme en Italie du Sud, l'inspiration ou l'initiation poétique d'Hésiode par les Muses, qui conforteraient les images religieuses, de sorte que la Vérité serait garantie par son origine divine, tandis que la perspective humaine se rapporterait aux opinions, exprimées par la sémantique du dokein (p.31-51). Or, en dépit de l'abondance des sources, l'A. passe sous silence un témoignage de Théophraste qui subvertit l'idée que Parménide accorderait une importance à la mort. Celui-ci aurait considéré le mort comme ressentant uniquement l'obscurité, le froid et le silence - ce qui exclut une doctrine du monde infernal ${ }^{8}$. Ce texte apparaît dans un contexte où s'insère le fr. B 16, par lequel l'A. achève son travail, en insérant la place de l'homme dans le cosmos (p. 198-209). Le recours aux images traditionnelles sur l'Hadès, les Tartares ou les données recueillies dans les nouveaux documents funéraires, projetées sur le Poème, me semblent forcer la narration.

8 Si la clé de la lecture de l'A. se trouve dans le « seuil » qui marque la rupture entre les deux zones, grâce à " une katabasis héroïque », on comprend que l'image du héros et du motif du char, analysés dans la perspective d'une initiation mystérique (p.71-75), devienne centrale. Or, comme le montre Aristote, l'initiation aux mystères n'enseigne rien, mais met le récipiendaire dans une disposition où il ressent des émotions, et conduit le profane à la Lumière 9 . Cela contraste avec l'initiation selon Parménide qui se rattache au savoir, ce qui atteste son originalité - comme l'admet l'A. Celle-ci contourne la difficulté en recourant aux modes des savoirs à cette époque. Cette étude constitue, mes yeux, l'apport le plus remarquable du livre. On y trouve les modes d'accès au monde infernal, les savoirs du devin et du prophète, du poète et du médecin, ainsi que « la tradition didascalique » qui s'accorde à l'enseignement (p. 86-91). 
Il est étonnant que les savoirs des sages et des philosophes soient à peine utilisés, et sans correspondance avec la critique parménidienne des doxai des mortels qui affrontent des problèmes philosophiques concernant le réel en devenir, notamment l'opposition entre les considérations des akrita phyla (qui sont les penseurs ioniens), et celles de penseurs qui accomplirent une trop grande séparation (krisis) dans le corps en distinguant lumière et obscurité (qui sont les pythagoriciens) ${ }^{10}$. S.R. attribue à Héraclite les opinions de la seconde partie, créant une confusion avec les akrita phyla. En fait, Parménide s'oppose tant à la sagesse (sophia) des physiologues présocratiques jusqu'aux sophistes, qu'au savoir qui aspire à la sagesse (philo-sophia) défendue par les Pythagoriciens, qui considèrent que seuls les dieux sont sages, les humains étant seulement des aspirants à la sagesse. D'ailleurs, le recours au pythagorisme s'accomplit dans le cadre de la table des opposés, avec la promotion de la lumière et de l'obscurité dans l'ordre physique, et jamais relativement à la religion (par l'immortalité de l'âme) ou même en invoquant les Muses, qui sont écartées du Poème. Bref, la portée critique du Poème n'est pas suffisamment mise en évidence, de sorte que les tentatives de Parménide de redresser tant la pratique du mythe que le caractère inintelligible du devenir grâce à la pensée, sont peu exploitées.

En fait, tout porte à croire que le proème est linéaire et concerne une initiation au savoir avec des épreuves. Il met en jeu un mythe d'origine et de genèse du savoir, qui anticipe par ses images et ses figures, la partie cosmogonique du Poème, déployée par un discours trompeur (apatèlon) mais converti, à la suite d'un redressement, en une cosmologie. C'est dans ce cadre que la pensée, fondée par l'Eon, accomplit elle-même un redressement, en convertissant les choses qui ne sont pas dans le présent (mè eonta) et qui sont absentes (apeonta) en choses présentes (pareonta) ${ }^{11}$. La pensée joue un rôle décisif dans la Poème ${ }^{12}$. Par sa continuité elle est proche de l'eon continu. Ce qui fait de l'eon, non pas le réel (quelle que soit la forme qu'on lui attribue, y compris une forme divine), mais la condition (toujours présente par son statut d'être toujours dans le présent) de la pensée, grâce à laquelle le savoir trouve sa légitimation. Rien dans cette démarche n'autorise une approche religieuse du Poème, même si les images religieuses permettent, par contraste, de conforter cette exclusion.

11 L'A., fascinée, non sans raison, par les nouveaux documents funéraires à sa disposition qu'elle voulut intégrer à tout prix dans le Poème de Parménide, perd de vue aussi bien le sens de la complexité archaïque que le fait que celle-ci n'a pu être ébranlée que par l'avènement de la question de la pensée, dont Parménide fut le premier théoricien. Je tiens néanmoins à souligner que ces critiques constructives n'amoindrissent en rien l'ingéniosité de la démarche de Sofia Ranzato, qui nous livre un nouveau visage de Parménide, et qui révèle ses potentialités heuristiques et scientifiques. Nous attendons avec intérêt la suite de ses recherches. 


\section{NOTES}

1. J'ai proposé une analyse critique plus exhaustive du livre sous le titre «En quête de la complexité parménidienne ", dans la Revue de philosophie ancienne 34 (2016).

2. Cette monographie a été publiée selon deux versions: Mythe et philosophie chez Parménide, Bruxelles, 1986, 1990² (que je citerai MPhP), et La pensée de Parménide, Bruxelles, $2008^{3}$ (que je citerai PP). Cf. MPhP, p 68.

3. Cf. La philosophie face à la question de la complexité. Le défi majeur du $21^{e}$ siècle, 2 vol., Bruxelles, 2014, où je traite de la complexité archaïque dans le volume 1, p. 166-304.

4. Cf. «Images, mythes, catalogues, généalogies et mythographies », Kernos 19 (2006), p. 11-21 et "Fécondité des pratiques catalogiques », ibid., p. 249-266, mais déjà « Genèse et structure dans le mythe hésiodique des races ", in F. BLAISE, P. JUDET DE LA COMBE, Ph. ROUSSEAU (éd.), Le métier du mythe, Lille, 1996, p. 475-518, ainsi que PP, p. 66-89.

5. Voir, en plus de la troisième édition de mon livre sur Parménide, mon étude « Émergence de la thématique de la providence divine : De Diogène d'Apollonie à Platon ", in P. D'HOINE, G. VAN RIEL (éd.), Fate, Providence and Moral responsability in Ancient, Medieval and Early modern thought. Studies in Honour of Carlos Steel, Leuven, 2014, p. 3-21.

6. Cf. G. PUGLIESE CARRATELLI, Les Lamelles d'or orphiques, Paris, 2003, qui traduit et complète l'édition italienne de 2001.

7. Voir aussi Orphicum et Orphicis similium testimonia et fragmenta (fr. 474-496) : «lamellae aureae », dans Poetae Epici Graeci. Testimonia et fragmenta, Pars II, Munich/Leipzig, par les soins de A. Bernabé. Sur une mise au point de cette problématique, voir Cl. CALAME, «Les lamelles funéraires d'or : textes pseudo-orphiques et pratiques rituelles », Kernos 21 (2008), p. 299-311.

8. Théophraste, De sensu, 3 (= fragments des Présocratiques de Diels-Kranz A 46, p. 246, 3-17).

9. Aristote, fr. 15 Ross (= Synésius, Dion). Cf. E. DES PLACES, La Religion grecque. Dieux, cultes, rites et sentiment religieux dans la Grèce antique, Paris, 1969, p. 213-214.

10. $P P$, p. 281 sq. et $422 s q$.

11. $P P$, p. $179-196$.

12. MPHP, p. 207-260 et PP, p. 343-403.

\section{AUTEURS}

\section{LAMBROS COULOUBARITSIS}

(Université libre de Bruxelles) 